\title{
A NKG2D Thr72Ala polymorphism is not associated with HAM/TSP and proviral load values in Peruvian HTLV-1 infected patients with HAM/TSP and asymptomatic carriers
}

\author{
Cynthia Bernia*, Jason Rosado, Giovanni López, Eduardo Gotuzzo, Michael Talledo \\ From 17th International Conference on Human Retroviruses: HTLV and Related Viruses \\ Trois Ilets, Martinique. 18-21 June 2015
}

\section{Background}

NKG2D is an activating receptor mainly expressed on Natural Killer (NK) cells, its ligands has an abnormal expression in virus infected cells and tumoral cells. The SNP rs2255336 G/A generates a substitution of alanine to threonine (NKG2D Thr72Ala) associated with a low and high cytotoxic activity of NK cells respectively. This study investigated the association between the NKG2D Thr72Ala polymorphism with HAM/TSP and PVL values in Peruvian HTLV-1 infected patients with HAM/TSP and asymptomatic carriers.

\section{Methods}

215 Peruvian HTLV-1 infected patients (142 asymptomatic carriers (AC) and $73 \mathrm{HAM} / \mathrm{TSP}$ ) were evaluated. NKG2D Thr72Ala polymorphism were genotyped by polymerase chain reaction using allele-specific primers, 37 ancestry informative markers (AIM) were genotyped to correct by population stratification using the first three principal components. Proviral load (PVL) was measured using the endogenous retrovirus 3 (ERV-3). Association of the NKG2D Thr72Ala polymorphism with HAM/TSP and PVL were performed by univariate analysis using Pearson's chi-square test and Mann-Whitney U-test and by multivariate analysis using logistic regression and linear regression analysis. STATA software was used for all analysis.

\section{Results}

The NKG2D Thr72Ala polymorphism with G/G genotype showed a higher prevalence in asymptomatic carrier
(64.13\%) respect to HAM/TSP patients (34.87\%), however these differences were not statistically significant $(P>0.05)$. No association was found between the different genotypes of NKG2D Thr72Ala with PVL (P > 0.05), or with the presence of HAM/TSP $(\mathrm{P}>0.05)$. The assessment of the association between allele $\mathrm{G}(\mathrm{OR}=2.3760$; $\mathrm{CI}=0.7744$ to 7.2901$)$ or the allele $\mathrm{A}(\mathrm{OR}=0.4209$; $\mathrm{CI}=0.1372$ to 1.2914 ) with the presence of HAM/TSP were not statistically significant $(\mathrm{P}>0.05)$.

\section{Conclusions}

The results of this study indicate the need to evaluate the association of polymorphisms in NKG2D gene with HAM/TSP and PVL in other independent populations of HTLV-1 infected individuals in order to verify an association of disease state with host genetic.

Published: 28 August 2015

doi:10.1186/1742-4690-12-S1-O37

Cite this article as: Bernia et al:: A NKG2D Thr72Ala polymorphism is not associated with HAM/TSP and proviral load values in Peruvian HTLV-1 infected patients with HAM/TSP and asymptomatic carriers. Retrovirology 2015 12(Suppl 1):037

\footnotetext{
* Correspondence: cynthiabernia@yahoo.com.pe

Inst de Med Trop Alexander von Humboldt, Univ. Peruana Cayetano Heredia, Lima, Peru
} 